\title{
Absence of in vitro Procoagulant Activity in Immunoglobulin Preparations due to Activated Coagulation Factors
}

\author{
Adriana E. Oviedo ${ }^{a}$ María E. Bernardi ${ }^{a}$ Hugo A. Guglielmone ${ }^{a}$ b María S. Vitali ${ }^{a}$ \\ a Área de Desarrollo de Productos y Procesos, Laboratorio de Hemoderivados, Universidad Nacional de Córdoba, Córdoba, Argentina; \\ b Departamento de Bioquímica Clínica (CIBICI-CONICET), Facultad de Ciencias Químicas, Universidad Nacional de Córdoba, Córdoba, \\ Argentina
}

\section{Keywords}

Intravenous immunoglobulin · Subcutaneous immunoglobulin - Procoagulant activities - Factor Xla . Thromboembolic events

\section{Summary \\ Background: Immunoglobulin (IG) products, including intravenous (IVIG) or subcutaneous (SCIG) immunoglob- ulins are considered safe and effective for medical ther- apy; however, a sudden and unexpected increase in thromboembolic events (TE) after administration of cer- tain batches of IVIG products has been attributed to the presence of activated coagulation factors, mainly factor Xla. Our aims were to examine the presence of enduring procoagulant activity during the manufacturing process of IGs, with special focus on monitoring factor Xla, and to evaluate the presence of in vitro procoagulant activity attributed to coagulation factors in different lots of IVIG and SCIG. Methods: Samples of different steps of IG pu- rification, 19 lots of IVIG and 9 of SCIG were analyzed and compared with 1 commercial preparation of IVIG and 2 of SCIG, respectively. Factors II, VII, IX, XI and XIa and non-activated partial thromboplastin time (NAPTT) were assayed. Results: The levels of factors II, VII, IX, X and XI were non-quantifiable once fraction II had been re-dissolved and in all analyzed lots of IVIG and SCIG. The level of factor Xla at that point was under the detec- tion limits of the assay, and NAPTT yielded values greater than the control during the purification process. In SCIG, we detected higher concentrations of factor Xla in the commercial products, which reached values up to 5 times higher than the average amounts found in the 9 batches produced by UNC-Hemoderivados. Factor Xla in}

commercial IVIG reached levels slightly higher than those of the 19 batches produced by UNC-Hemoderivados. Conclusion: IVIG and SCIG manufactured by UNCHemoderivados showed a lack of thrombogenic potential, as demonstrated not only by the laboratory data obtained in this study but also by the absence of any reports of TE registered by the post marketing pharmacovigilance department.

(c) 2015 S. Karger GmbH, Freiburg

\section{Introduction}

Immunoglobulin (IG) products, including intravenous (IVIG) or subcutaneous (SCIG) immunoglobulins, are prepared from pools of human plasma obtained from at least 1,000 individual donors. These products are licensed for treatment of primary and secondary immunodeficiency disorders and some autoimmune and inflammatory diseases [1]. There are substantial differences in the manufacturing process of the IGs; for this reason, these products may vary in their concentration, osmolality, sugar components, sodium content, amino acids and other stabilizing agents as well as in their strategies for viral inactivation [2]. Overall, IG use is considered safe and effective, but common mild to moderate adverse events, including low-grade fever, headache, malaise, nausea, myalgia and urticaria, have been reported [3]. However, with the increasing use of these IGs, more severe side effects (mainly with IVIG) such as acute renal tubular necrosis, aseptic meningitis, and thromboembolic events (TE) have been described [4]. These last manifestations occur at a frequency of $2-3 \%$ and are most frequently acute, occurring either during the administration of the

\section{KARGER \\ Fax +497614520714

\section{(ㄷ) 2015 S. Karger GmbH, Freiburg}

1660-3796/15/0426-0397\$39.50/0 
IGs or within the following $24 \mathrm{~h}$. Underlying risk factors of the recipient (advanced age, thrombophilic status) and product factors (dosage, infusion rates, manufacturing process) are the most important matters to be considered as triggering events. In 2010, a sudden and unexpected increase of TE after administration of certain batches of an IVIG product was attributed to the presence of activated coagulation factors, mainly factor XIa (FXIa) [5]. FXI and immunoglobulins co-purify together; thus additional steps to remove contaminating traces of FXI [6] or an appropriate pasteurization process are required [7]. A recent report from the European Medicines Agency (EMA) informed that the main cause of TE associated with the use of some batches of IVIG is the presence of increased amounts of fXIa in these products [8]. Despite the relatively limited role of FXI in hemostasis, there are increasing evidences that this protease contributes to the development of thromboembolism in humans. Sustained thrombin generation through FIX activation by FXIa may potentiate several pro-thrombotic processes that result in a two-fold increase of the risk of developing TE [9]. Since 1997, the IG products manufactured by UNC-Hemoderivados are obtained from fraction II of Cohn-Oncley cold ethanol fractionation, which is produced with material from a pool of plasma donors. This process includes ultrafiltration, pasteurization, disaggregation, and formulation of the final products for intravenous use and pasteurization and formulation of subcutaneous presentations.

These data and the recent recommendations of the European Pharmacopeia prompted us to conduct this study. Our aims were i) to examine the presence of residual procoagulant activity during the IG manufacturing process, with special focus on FXIa monitoring and ii) to evaluate the presence of 'in vitro' procoagulant activity attributed to coagulation factors in different lots of IVIG and SCIG manufactured from 2010 to 2013.

\section{Material and Methods}

\section{Materials}

Samples (marked in bold) of the different steps of the IG purification process (fig. 1) were obtained from routine production of seven distinct lots manufactured between 2010 and 2013, and assayed either immediately or kept frozen at $-20{ }^{\circ} \mathrm{C}$ for no more than 2 months.

19 lots of IVIG and 9 of SCIG, manufactured between 2010 and 2013 in UNC-Hemoderivados, were analyzed and compared with one commercial preparation of 5\% IVIG and two of $16 \%$ SCIG, respectively. All samples were within their labeled shelf life.

\section{Quantitative Determination of the Activity of Coagulation Factors}

The activity of FII, FVII, FIX, and FXI was determined with one-stage clotting assays using deficient plasma from Trinity Biotech (Wicklow, Ireland) and an ACL 7000 coagulometer. For each factor, a working reference curve was arranged with a calibrator plasma and dilution buffer. Samples were tested in duplicate, and the results were expressed in IU/ml. The ACL 7000 was used in the mode 'low calibration curve' in order to ensure that the values fell within the readable range of the work curve obtained with the reference plasma. Non-quantifiable results were expressed in that manner when the values obtained ranged between 0.02 and $0.015 \mathrm{IU} / \mathrm{ml}$ (depending of the factor), which corresponded to the concentration of the lower point of the assay calibration curve.

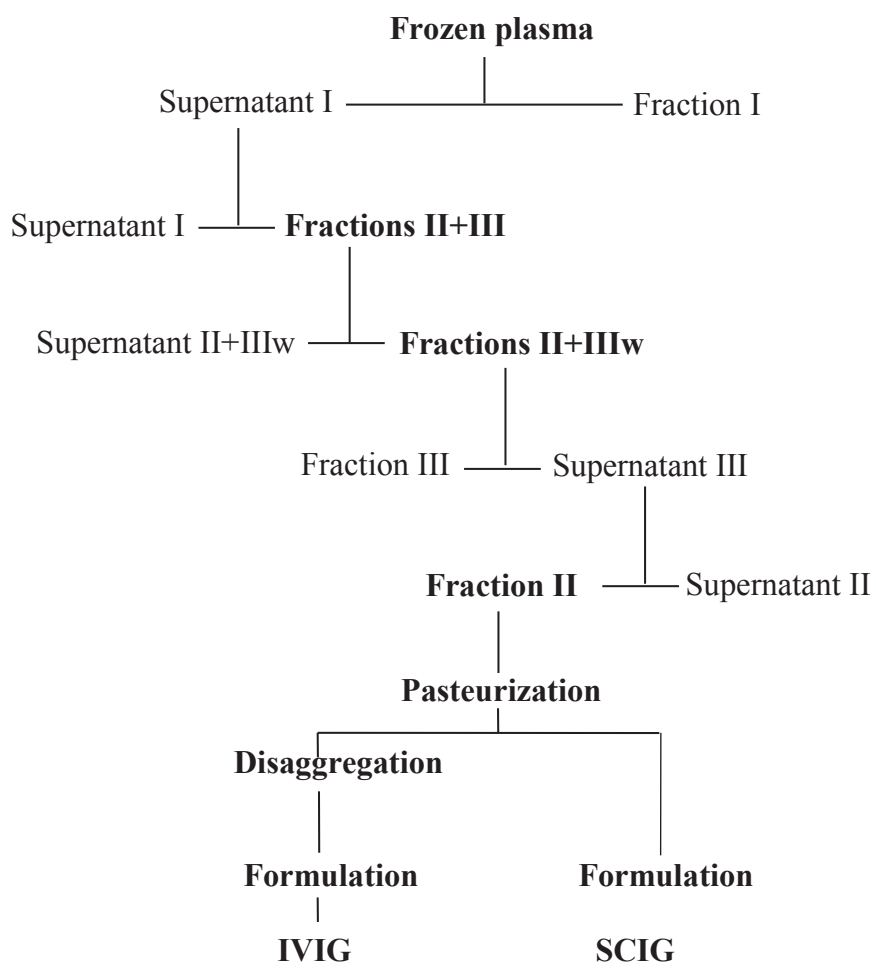

Fig. 1. Flow chart of the different steps of the IG purification process.

Quantitative Determination of FXIa

FXIa was assayed with a Biophen ${ }^{\circledR}$ chromogenic assay. In this assay, the FXIa present in the tested sample was assessed by the ability to activate FIX to FIXa, which forms an enzymatic complex with FVIIIa, also supplied in excess. This complex activates FX to FXa; the colored product obtained with a specific FXa substrate is directly related to the amount of FXIa to be measured. The released pNA was measured at $405 \mathrm{~nm}$ and the results were expressed in $\mathrm{ng} / \mathrm{ml}$. The calibrator provided in the kit was used to obtain the calibration curve, yielding values that ranged between $\sim 5$ and $\sim 0.625 \mathrm{ng} / \mathrm{ml}$. Non-quantifiable results were expressed as $<0.10 \mathrm{ng} / \mathrm{ml}$, which corresponded to the detection limit calculated by the validation method obtained from 5 assays. Sample blanks were carried out in all assays in order to assure the specificity of the method.

Determination of Non-Activated Partial Thromboplastin Time (NAPTT)

NAPTT is a coagulation assay sensitive to the presence of activated coagulation factors, according to the European Pharmacopoeia (Ph. Eur.)(2.6.22). The samples were diluted $1 / 10$ and $1 / 100$ in buffer solution. Human normal plasma was mixed with rabbit brain cephalin and incubated at $37^{\circ} \mathrm{C}$; afterwards, the sample dilutions and $\mathrm{CaCl}_{2}$ were added, and the clotting time was measured. As control, buffer solution was included in the same run of tests. The results were expressed as mean of the duplicates, in seconds, for each dilution assayed. The test was considered valid when the coagulation time measured for the control tube (which contained buffer assays instead of sample material) was 200-320 s and the values of the samples yielded results longer than $150 \mathrm{~s}$.

\section{Statistics}

The results are expressed as mean \pm SEM.

\section{Results}

Samples of 7 batches obtained from 7 different steps of the purification process of IGs were obtained, and the coagulation factors were assayed (table 1). 
Fig. 2. Levels of FXIa during the IG manufacturing process.

Table 1. Levels of the coagulation factors in the different steps of the purification process

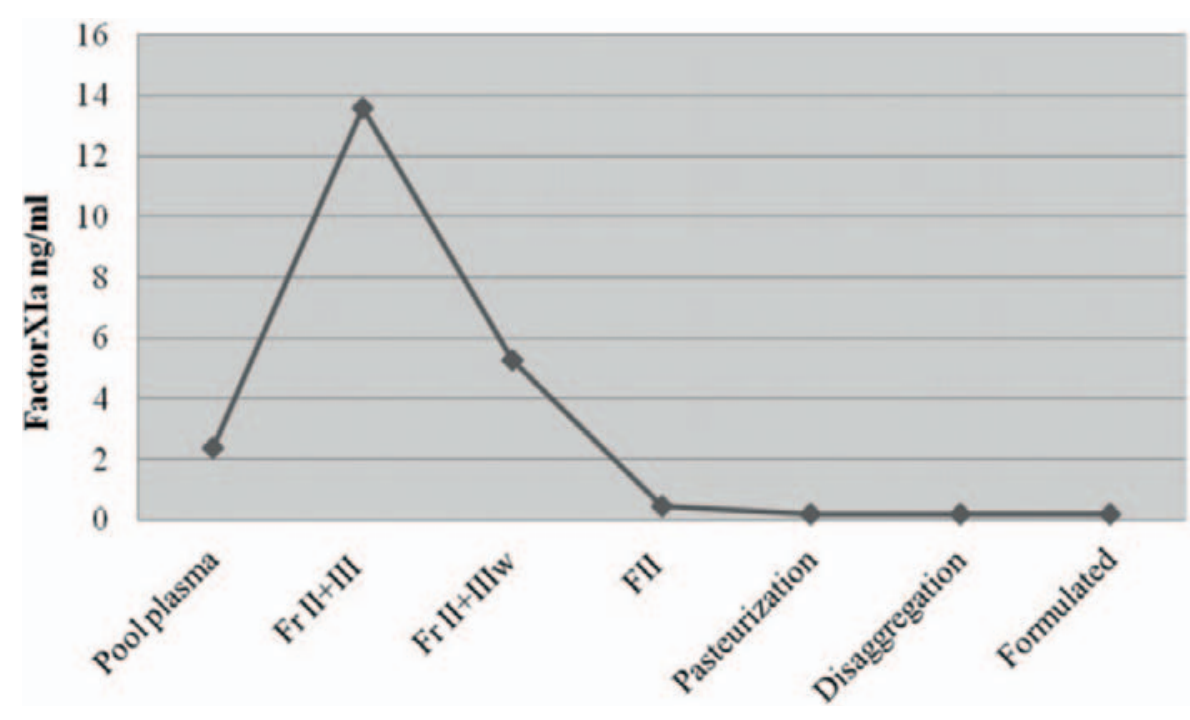

\begin{tabular}{llllll}
\hline Samples $(\mathrm{n}=7)$ & $\mathrm{IU} / \mathrm{ml}$ & & & \\
\cline { 2 - 6 } & factor II & factor VII & factor IX & factor X & factor XI \\
\hline Plasma pool & $0.79 \pm 0.30$ & $1.03 \pm 0.30$ & $0.72 \pm 0.31$ & $1.20 \pm 0.40$ & $0.98 \pm 0.30$ \\
Fractions II+III & $0.87 \pm 0.40$ & $3.09 \pm 0.40$ & $1.48 \pm 0.23$ & $0.51 \pm 0.11$ & $1.02 \pm 0.40$ \\
Fractions II+IIIw & $0.65 \pm 0.40$ & $2.95 \pm 0.70$ & $1.04 \pm 0.54$ & $0.04 \pm 0.01$ & $0.87 \pm 0.40$ \\
Fraction II & $<0.015$ & $0.07 \pm 0.02$ & $<0.015$ & $<0.015$ & $0.07 \pm 0.02$ \\
Pasteurization & $<0.015$ & $<0.015$ & $<0.015$ & $<0.015$ & $<0.015$ \\
Disagreggation* & $<0.015$ & $<0.015$ & $<0.015$ & $<0.015$ & $<0.015$ \\
Formulation & $<0.015$ & $<0.015$ & $<0.015$ & $<0.015$ & $<0.015$ \\
\hline *Orn & & & & \\
\hline
\end{tabular}

The results of FXIa and NAPTT are shown in figure 2 and table 2, respectively. Both assays showed a reduction in the thrombogenic potential along with the progress of IG production. In fact, the level of FXIa showed an initial increase of fractions II + III followed by a significant decrease during the subsequent steps of the process. Meanwhile, NAPTT $1 / 10$ and 1/100 behaved in the same way, with the exception that the maximum thrombogenic activity was observed in fractions II + IIIw, compared to the control time (91 \pm 7 s vs. $285 \pm 19$ s).

The levels of the coagulation factors in 19 IVIG and 9 SCIG, including commercial commodities (1 for IVIG and 2 for SCIG) were assayed. The levels of FII, FVII, FIX, FX, and FXI were nonquantifiable in the IVIG presentations of all analyzed products (19 lots and one commercial product) (data not shown). The concentration levels of FXIa (fig. 3a) and NAPTT for IVIG are shown in figure 3b. FXIa in commercial IVIG reached levels slightly higher than the average levels of the 19 batches produced by UNC-Hemoderivados. NAPTT $1 / 10$ and $1 / 100$ yielded similar values in all samples analyzed.

In SCIGs, the levels of FII, FVII, FIX, FX, and FXI were also non-quantifiable in all lots produced in UNC-Hemoderivados. Similar results were found for FII, FVII, FIX, and FX in the 2 commercial products analyzed; however, an considerable increase of the concentration of FXI $(0.95$ and $1.98 \mathrm{IU} / \mathrm{ml})$ was detected, which represents an increase of 7 times the average value of this
Table 2. NAPTT during the production process of IGs

\begin{tabular}{lcl}
\hline Samples $(\mathrm{n}=7)$ & NAPTT $1 / 10, \mathrm{~s}$ & NAPTT $1 / 100, \mathrm{~s}$ \\
\hline Plasma pool & $116 \pm 12$ & $253 \pm 46$ \\
Fractions II+III & $116 \pm 9$ & $245 \pm 23$ \\
Fractions II+IIIw & $91 \pm 7$ & $237 \pm 12$ \\
Fractions II & $304 \pm 40$ & $333 \pm 54$ \\
Pasteurization & $297 \pm 32$ & $311 \pm 39$ \\
Disaggregation & $318 \pm 44$ & $327 \pm 49$ \\
Formulation & $319 \pm 38$ & $328 \pm 40$ \\
Control tube & $285 \pm 19$ & $289 \pm 19$ \\
\hline *Only for IVIG products. & & \\
\hline
\end{tabular}

factor in products manufactured by UNC-Hemoderivados (data not shown). Figure 4 shows the values of FXIa (a) and NAPTT (b) in the lots of SCIG. We detected higher concentrations of FXIa in the commercial products, which reached values up to 5 times the average values found in the 9 batches produced in UNC-Hemoderivados. In fact, the values of FXIa ranged from 0.10 to $4 \mathrm{ng} / \mathrm{ml}$ but always remained lower than the levels detected in the two commercial products. For NAPTT, all levels were higher than the those recommended by the European Pharmacopeia in all batches produced by UNC-Hemoderivados. The two commercial product presented values lower than $150 \mathrm{~s}$, suggesting a high potential thrombogenic risk. 


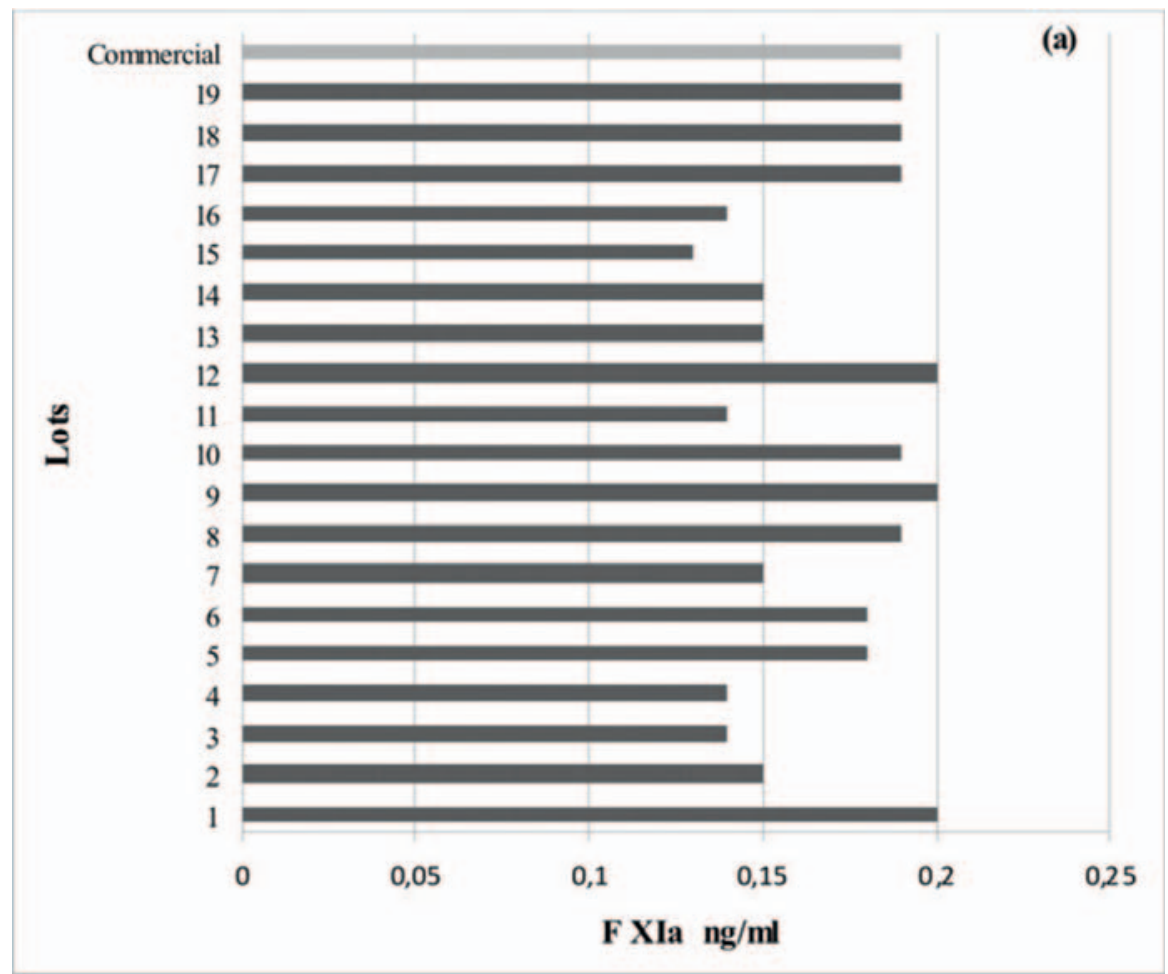

Fig. 3. Concentration of FXIa (a) and NAPTT (b) in IVIG preparations.

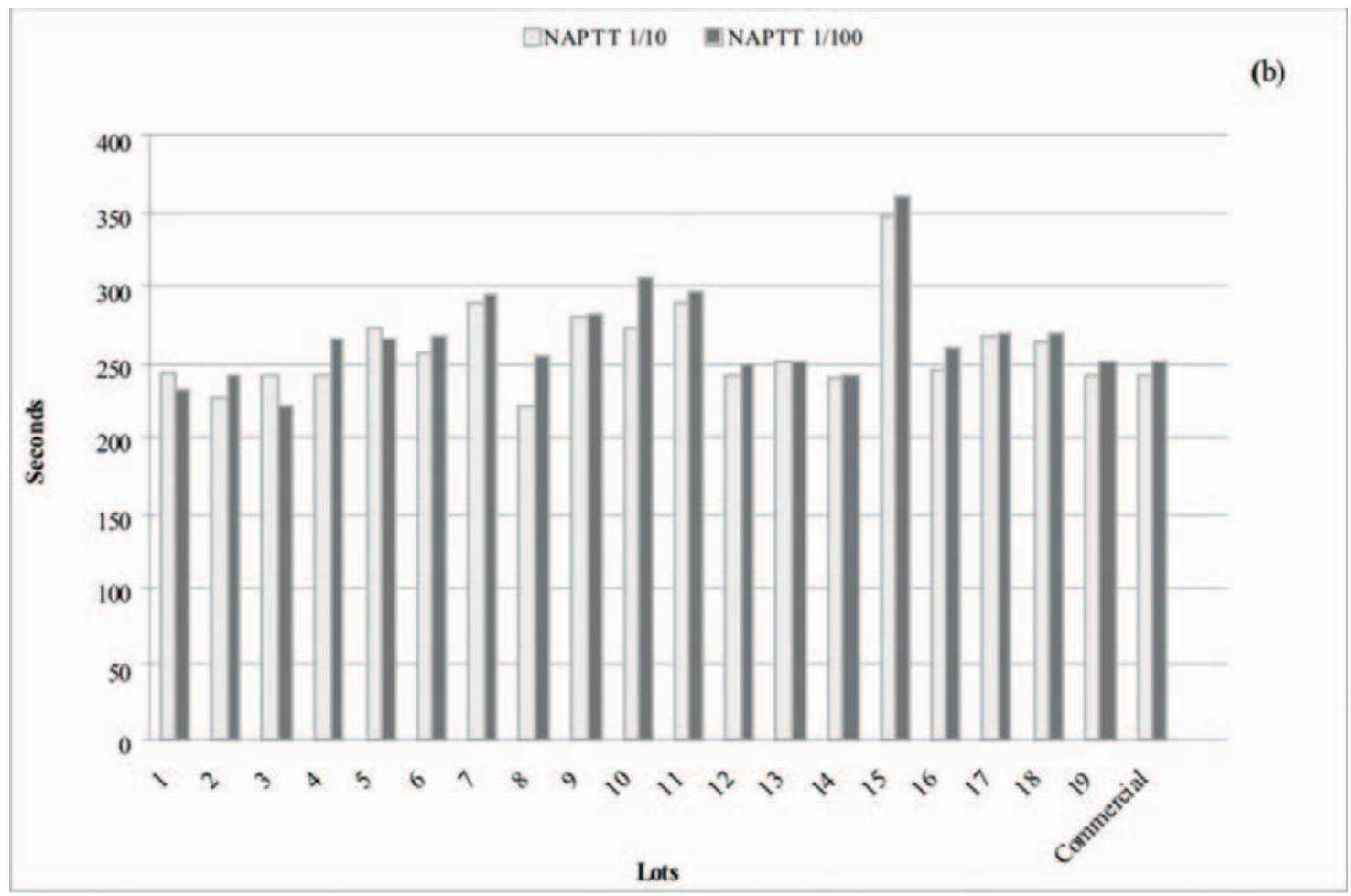

\section{Discussion}

From a commercial point view, the Cohn-Oncley cold ethanol fractionation [11] is the most frequently used method for plasma fractionation in the large-scale purification of plasma components, such as IG and albumin products. The original process has under- gone considerable modifications over time in order to improve the purity and safety of the products or to isolate determined plasma components. Despite these changes, remaining contaminant proteins present in the IG fractions may affect the quality, safety, and efficacy of the products. In fact, the recently revised Ph. Eur. Monograph on human normal immunoglobulin for intravenous ad- 


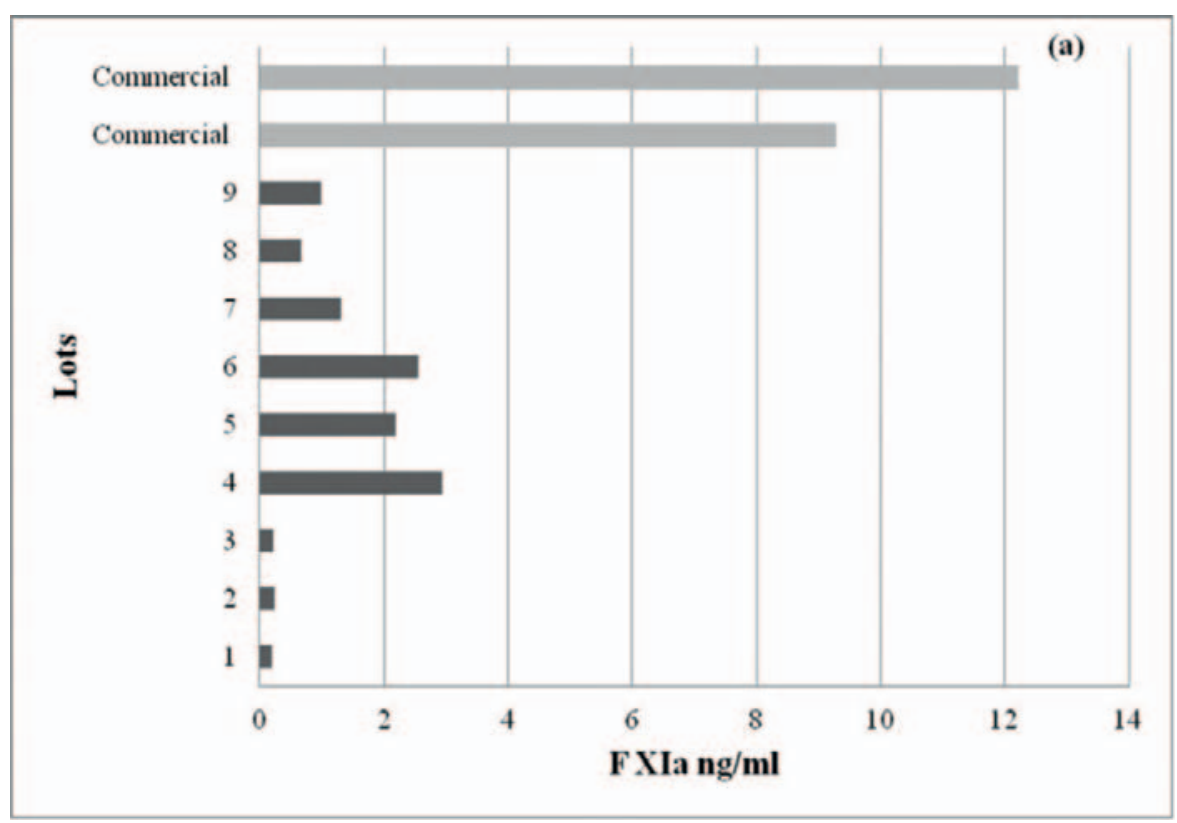

NAPTT $1 / 10(s) \quad$ NAPTT $1 / 100(s) \quad$ (b)

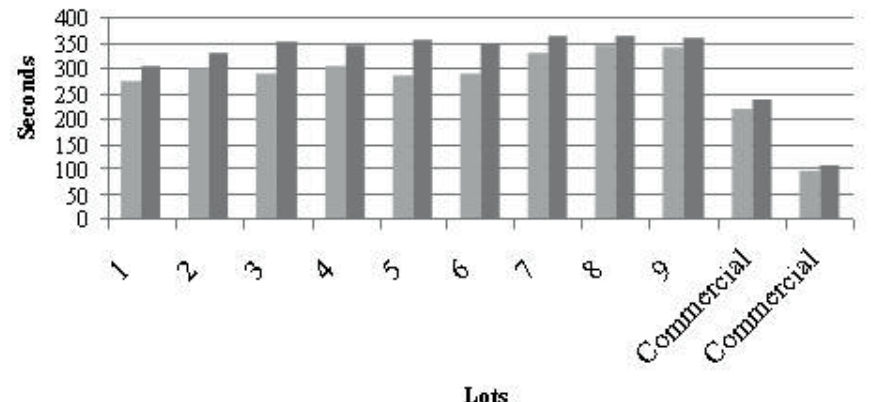

Fig. 4. Concentration of FXIa (a) and NAPTT (b) in SCIG preparations.

Lots

ministration requires that the production of IG process includes steps that remove thrombosis-generating agents (coagulation factors and/or zymogens) which mainly catalyze activation of FXI to FXIa [12]. The same recommendation is given in the Ph. Eur. Monograph for SCIG production since the raw material and part of the purification process are similar to IVIG manufacturing [13].

In this study, we systematically investigated the presence of coagulation factors with special emphasis on FXIa during the whole IG purification procedure, as well as in the formulated IVIG and SCIG products. Cold ethanol fractionation has been mostly used for IG production by our group, including some modifications in order to improve the purity and viral safety. Our results showed a lack of thrombogenic material from clotting factors in the different steps of the process. In fact, there is a noticeable drop in the concentration of clotting FII, FVII, FIX, FX, and FXI from fraction II, which reached levels below the limits of quantification in those steps that follow IVIG pasteurization, disaggregation, and formulation. These results were predictable since clotting factors are unlikely to be found at this industrial level of production, and only a very low concentration of FVII $(0.17 \mathrm{IU} / \mathrm{ml})$ and FXI $(0.07 \mathrm{IU} / \mathrm{ml})$ were found on fraction II. Similar results have been observed by José et al. [14] during the purification process of IVIG Flebo- gamma ${ }^{\circledR}$ DIF. The global test NAPTT was used to determine the presence of activated coagulation factors, allowing for detection of an important prolongation of the coagulation time in fraction II and the subsequent steps of the manufacturing process. These results were in accordance with the levels of FXIa since the NAPTT test is highly sensitive to this factor [15]. Indeed, a dramatic drop in the levels of FXIa below the detection limits of the assay was accompanied by an important prolongation of NAPTT. Both assays clearly demonstrated a high procoagulant activity of fractions II+IIIw and undetectable actions in fraction II as well as after pasteurization, disaggregation and the formulation process.

Our results demonstrated very low levels of coagulation FII, FVII, FIX, FX and F,XI in the different batches of IVIG in both, commercial $(n=1)$ and UNC-Hemoderivados products $(\mathrm{n}=19)$. The presence of FXIa in all the 19 IVIG lots analyzed yielded values $\leq 0.2 \mathrm{ng} / \mathrm{ml}$ and the commercial product presented an increase levels slightly higher than the average values of the UNC-Hemoderivados products. However, this increase did not represent a potential thrombogenic load since the level of FXIa found in lots responsible for TE yielded results close to $47 \mathrm{ng} / \mathrm{ml}$ [16]. On the other hand, when NAPTT rates were analyzed in all batches of our IVIG products and in those of commercial sources, all results were 
above the control time, which clearly indicates a lack of thrombogenic potential. These results also allowed us to infer that there is a lack of correlation between NAPTT and FXIa when present in very low concentrations. In fact, it has been recently reported that the NAPTT test has satisfactory sensitivity for detection of levels $\geq 1 \mathrm{ng} / \mathrm{ml}$ of FXIa [10].

The results of the SCIG analysis showed a profile similar to IVIG. The levels of FII, FVII, FIX, and FX were very low in both UNC-Hemoderivados $(n=9)$ and commercial $(n=2)$ products, but an important difference in the level of FXI was noted. In fact, this factor presented rates that ranged from 1 to $2 \mathrm{IU} / \mathrm{ml}$ in the commercial products compared to $<0.015 \mathrm{IU} / \mathrm{ml}$ observed in the UNC-Hemoderivados batches. This result can be correlated with the levels of FXIa obtained in the same batches of SCIG analyzed. Indeed, an increase of almost 5 times (expressed in $\mathrm{ng} / \mathrm{ml}$ ) was found in the commercial products, compared to those manufactured in UNC-Hemoderivados. FXI is the zymogene of the active enzyme FXIa, and this conversion may increase during the storage of the plasma source used for IG manufacturing at $4^{\circ} \mathrm{C}$ [17]. We observed considerable variations in the levels of FXIa in our SCIG batches, but these were always lower than values of the commercial products. Similarly, the levels of FXIa also demonstrated a correlation with NAPTT. In fact, we observed that one of the commercial lots with a high concentration of FXIa showed a lower value of NAPTT. In contrast, another batch that showed a low concentration of this factor presented NAPTT levels similar or above that of the control tube.
Despite the large number of companies that produce plasma derivatives in the world, only a few studies have been conducted about the thrombogenic potential of the IG during the production process and the resulting products. The IVIG and SCIG manufactured in UNC-Hemoderivados did not have any thrombogenic potential, as demonstrated not only by the laboratory data obtained in this study but also by the fact that there are no reports of TE registered by the Post marketing Pharmacovigilance Department. Nonetheless, after the publication of some reports about the development of TE after administration of products derived from purification of IG, some precautions should be taken in order to avoid this serious event that may be harmful for human health. In this regard, in our production process, the thrombogenic potential of IG products is determined by our team, even when it is not required by the regulatory authorities. Besides, an addition of known amounts of FXIa in different steps of the manufacturing process of IGs is being carried out to determine at what point of our production process these procoagulant factors are currently being eliminated.

\section{Disclosure Statement}

The authors are salaried employees of UNC-Hemoderivados.

\section{References}

1 Katz U, Achiron A, Sherer Y, Shoenfeld Y: Safety of intravenous immunoglobulin (IVIG) therapy. Autoimmun Rev 2007;6:257-259.

2 Buchacher A, Iberer G: Purification of intravenous immunoglobulin $\mathrm{G}$ from human plasma-aspects of yield and virus safety. Biotechnol J 2006;1:148-163.

3 Daniel G, Menis M, Sridhar G, Wallace A, Golding B, Anderson S, Martin D, Ball R, Izurieta H: Immune globulins and thrombotic adverse events as recorded in a large administrative database in 2008 through 2010. Transfusion 2012;52:2113-2121.

4 Rajabally Y, Kearney D: Thromboembolic complications of intravenous immunoglobulin therapy in patients with neuropathy: a two-year study. J Neurol Sci 2011;308:124-127.

5 Seifner A, Beck G, Eichmeir S, Lackner F, Weber K, Wolleim G: Assessment of immunoglobulin concentrates on thrombogenic activity by thrombin generation assay, prekallikrein activator assay, and size-exclusion chromatography. Transfusion 2014;54:376-383.

6 Bouma B, Griffin J: Human blood coagulation factor XI: purification, properties, and mechanism of activation by activated factor XII. J Biol Chem 1977;252: 6432-6437.
7 Marzo J, Bono R, Carretero M, Lopez M, Ristol P, Jorquera J: Pasteurization inactivates clotting enzymes during Flebogamma ${ }^{\circledR}$ and Flebogamma ${ }^{\circledR}$ DIF production. Webmed Central Immunother 2011; 1:WMCOO1425.

8 European Medicines Agency: European Medicines Agency recommends lifting of suspension of Octagam. Recommendation follows implementation of improved manufacturing process and other preventive measures. www.ema.europa.eu/docs/en_GB/document_library/ Press_release/2011/04/WC500105247.pdf (last accessed September 9, 2015).

9 Smith S, Gailani D: Update on the physiology and pathology of factor IX activation by factor XIa. Expert Rev Hematol 2008;1:87-98.

10 Komenda M, Stadler D, Malinas T, Pragst I, Schmutz P, Minnig K, El Menyawi I: Assessment of the ability of the Privigen ${ }^{\circledR}$ purification process to deplete thrombogenic factor XIa from plasma. Vox Sang 2014;107:26-36.

11 Cohn EJ, Oncley JL, Strong LE, Hughes WL, Armstrong SH: Chemical, clinical, and immunological studies on the products of human plasma fractionation. I: the characterization of the protein fractions of human plasma. J Clin Invest 1944;23:417-432.

12 European Pharmacopeia 7.0. PA/PH/ exp. 6B/T (11) 14 PUB. Human normal immunoglobulin for intravenous administration: 2012 Jan. Report No.:01/2012:0918.
13 European Pharmacopeia Online 7.6. Human normal immunoglobulin for intravenous administration. Monograph 0338. Council of Europe, Strasbourg, France, 2013.

14 José M, Marzo N, Pons B, Herrerias A, López M, Faro Merche, López M, Jorquera JI: Flebogamma ${ }^{\circledR}$ DIF (intravenous immunoglobulin) purification process effectively eliminates procoagulant activities. Biological 2013;6:393-399.

15 Ma L, Sun P, Lin FZ, Diao G, Li CQ: Quantitative determination of activated factor XI as an impurity in therapeutic immunoglobulins from Chinese blood fractionation companies. Genet Mol Res 2013;3:25562561

16 Gray E: NIBSC Investigation on Thrombogenicity Test for IVIG. Risk Mitigation Strategies to Address Potential Procoagulant Activity in Immune Globulin Products Presentations. FDA, U.S. Food and Drug Administration. Department of Health \& Human Services, 2011. www.fda.gov/downloads/BiologicsBloodVaccines/ NewsEvents/WorkshopsMeetingsConferences/ UCM260790.pdf (last accessed September 9, 2015).

17 Hoem NO, Johannsen S, Brisud K: Formation of an association between factor XII and kallikrein in human plasma: significance of storage of plasma and the functional state of kallikrein. Thromb Res 1992;66:421-434. 